\title{
Revalidação do painel sorológico positivo para sífilis - uma ferramenta no controle da qualidade de kits para diagnóstico da sífilis
}

\section{Revalidation of the positive serological panel for syphilis - a tool in the quality control of kits for syphilis diagnosis}

\section{Danielle Custódio Deslandes do Passo',1I,* iD \\ Marisa Adati Coelho' \\ Danielle Copello Vigo' \\ Álvaro da Silva Ribeiro' (D) \\ Roberto Machado do Passo' iD \\ Helena Pereira da Silva Zamith" iD}

\section{RESUMO}

Introdução: A sífilis é uma doença infecciosa de evolução lenta causada por uma bactéria Gramnegativa do grupo das espiroquetas, exclusiva do ser humano, chamada Treponema pallidum. No combate da sífilis, uma importante ferramenta é a análise laboratorial através de produtos para diagnóstico in vitro que permite o diagnóstico da doença. A eficácia destes produtos é avaliada frente a painéis sorológicos compostos por amostras verdadeiro positivas e negativas. Objetivo: Reavaliar a reatividade das 153 amostras que constituem o painel sorológico positivo para sífilis do Laboratório de Sangue e Hemoderivados (LSH) do Instituto Nacional de Controle de Qualidade em Saúde (INCQS). Método: Revalidação do painel sorológico para sífilis através de análise retrospectiva de dados laboratoriais do LSH do período de 2011 a 2015 atendendo aos critérios de positividade em cinco metodologias diferentes e volume de amostra $\geq 10 \mathrm{~mL}$. Resultados: Das 172 amostras iniciais, 153 apresentaram volume de estoque $\geq 10 \mathrm{~mL}$. No período proposto foram identificados 46 produtos para diagnóstico da sífilis com laudo satisfatório compreendendo cinco metodologias diferentes. Os resultados analíticos das 153 amostras pertencentes ao painel positivo foram analisados nos protocolos de registro de resultados destes produtos. Após a reavaliação da reatividade das 153 amostras do painel positivo, 130 foram revalidadas como positivas enquanto 23 foram consideradas como indeterminadas. Conclusões: O painel positivo para sífilis revalidado permanecerá sendo instrumento essencial na análise prévia para fins de regularização dos kits para diagnóstico da doença.

PALAVRAS-CHAVE: Sífilis; Painel Sorológico; Controle de Qualidade

\section{ABSTRACT}

Introduction: Syphilis is a slowly evolving infectious disease caused by a gram-negative bacterium in the group of spirochetes, exclusive to humans, called Treponema pallidum. In the fight against syphilis, an important tool is the laboratory analysis through products for in vitro diagnosis that allows the diagnosis of the disease. The effectiveness of these products is assessed against serological panels composed of true positive and negative samples. Objective: To reassess the reactivity of the 153 samples that make up the positive serological panel for syphilis at the Laboratory of Blood and Blood Products (LSH) of the National Institute for Quality Control in Health (INCQS). Method: Revalidation of the serological panel for syphilis through retrospective analysis of LSH laboratory data from 2011 to 2015, meeting the positivity criteria in 5 different methodologies and sample volume $\geq 10 \mathrm{~mL}$. Results: Of the 172 initial samples, 153 had stock volume $\geq 10 \mathrm{~mL}$. In the proposed period, 46 syphilis diagnosis products were identified with a satisfactory report, comprising 5 different methodologies. The analytical results of the 153 samples belonging to the positive panel were analyzed in the protocols for recording the results of these products. After re-evaluating the reactivity of the 153 samples from the positive panel 130, they were revalidated as positive while 23 were considered to be indeterminate. Conclusions: The positive panel for revalidated syphilis will remain an essential instrument in the previous analysis for the purpose of regularizing kits for diagnosing the disease.

KEYWORDS: Syphilis; Serological Panel; Quality Control 


\section{INTRODUÇÃO}

A sífilis é uma doença infecciosa de evolução lenta causada por uma bactéria Gram-negativa do grupo das espiroquetas, Treponema pallidum, que é exclusiva do ser humano. É uma enfermidade sistêmica, pois acomete praticamente todos os órgãos e sistemas. Esta doença desafia há séculos a humanidade e, apesar de ser curável através de um tratamento eficaz e de baixo custo, permanece como um grave problema de saúde pública até os dias atuais ${ }^{1}$. De acordo com dados da Organização Mundial de Saúde (OMS), a sífilis atinge anualmente aproximadamente 5,6 milhões de pessoas em todo mundo, sendo $90 \%$ destes casos nos países em desenvolvimento ${ }^{2,3}$. Os dados epidemiológicos da doença no Brasil presentes no Boletim Epidemiológico Sífilis 2018 do Ministério da Saúde, entre os anos de 2010 e 2017, revelam uma elevação significativa nas taxas de detecção da sífilis congênita, em gestantes bem como da sífilis adquirida ${ }^{4}$.

A principal via de transmissão da doença é o contato sexual (sífilis adquirida), seguida pela transmissão vertical da mãe para o filho ocorrida durante a gestação (sífilis congênita) $)^{5,6,7}$. A transmissão da sífilis por transfusão sanguínea, embora possível, tornou-se rara devido à triagem sorológica obrigatória de doadores quanto à presença de agentes infecciosos como vírus da imunodeficiência humana (HIV) $1 / 2$, vírus linfotrópico de células T humanas (HTLV)-1/2, vírus da hepatite B (HBV), vírus da hepatite C (HCV), Trypanosoma cruzi e $T$. pallidum $^{8,9}$. Aliado a isto, o pouco tempo de sobrevivência da bactéria fora do organismo humano, especialmente em baixas temperaturas como as usadas para a conservação das bolsas de sangue, contribui para diminuição da transmissão da doença pela transfusão sanguínea ${ }^{10}$.

A análise laboratorial de amostras biológicas através de produtos para diagnóstico in vitro é uma importante ferramenta no combate à sífilis, permitindo o diagnóstico e, consequentemente, o tratamento adequado e o monitoramento da resposta ao tratamento. Além disso, a utilização destes produtos na triagem sorológica das bolsas de sangue propicia o controle da transmissão da doença. O controle da qualidade destes produtos é realizado através de análise prévia, atividade efetuada para verificar características do produto com finalidade de registro, alteração (quando couber) ou revalidação sendo um requisito obrigatório preconizado pela Agência Nacional de Vigilância Sanitária (Anvisa) para comercialização e utilização desses produtos no Brasil ${ }^{11}$. Esta análise prévia é realizada pelo Laboratório de Sangue e Hemoderivados (LSH) do Departamento de Imunologia do Instituto Nacional de Controle de Qualidade em Saúde (INCQS). No controle da qualidade dos kits para diagnóstico é verificada a conformidade do produto em relação a dois parâmetros distintos: sensibilidade e especificidade clínica ou diagnóstica. A sensibilidade clínica é avaliada pela incidência de resultados verdadeiramente positivos obtidos quando o teste é aplicado em indivíduos sabidamente portadores da doença em questão. A especificidade clínica é avaliada pela incidência de resultados verdadeiramente negativos obtidos quando o teste é aplicado em indivíduos sabidamente não portadores da doença em questão ${ }^{11}$. Um dos instrumentos utilizados para avaliação destes dois parâmetros são os painéis sorológicos definidos como um conjunto de amostras produzidas a partir de plasma humano processado. As amostras que contêm determinantes antigênicos de um determinado marcador constituem o painel sorológico positivo e as que não contêm constituem o painel sorológico negativo ${ }^{12}$. Desta forma, os painéis constituídos e empregados na análise de produtos são de extrema relevância e a sua revalidação, com critérios claramente definidos, asseguram a robustez e consistência necessárias a este instrumento.

Sendo assim o objetivo deste trabalho foi revalidar o painel sorológico positivo para sífilis do LSH, instrumento utilizado na avaliação da sensibilidade dos kits para o diagnóstico da sífilis.

\section{MÉTODO}

O painel sorológico constituído de 172 amostras verdadeiramente positivas para sífilis foi confeccionado a partir de unidades de plasma provenientes de serviços de hemoterapia das diferentes regiões do país. As amostras com volume superior a $200 \mathrm{~mL}$, recebidas pelo LSH/INCQS entre os anos de 1996 e 2006, foram caracterizadas cumprindo rigorosamente a legislação vigente com o emprego de testes treponêmicos que detectam anticorpos específicos contra T. pallidum como: Enzyme Linked Immunosorbent Assay (ELISA), teste rápido imunocromatográfico (TR), imunofluorescência indireta (IFI) e testes não treponêmicos como Venereal Diseases Research Laboratory (VDRL) e Rapid Plasm Reagin (RPR) ${ }^{9,13}$.

O procedimento empregado para a revalidação das amostras do painel sorológico positivo para sífilis consistiu numa análise retrospectiva dos resultados laboratoriais do LSH/INCQS obtidos nos kits para diagnóstico da sífilis, avaliados no período de janeiro de 2011 a dezembro de 2015, cujos laudos de análise (LA) foram satisfatórios. Foram identificados os protocolos de registro de resultado de todas as amostras do painel referente a cada um dos produtos analisados. Para a análise dos resultados, foi elaborada uma planilha em $\operatorname{Excel}^{\circledR}$ contendo a identificação do kit, a amostra e os resultados analíticos nas diferentes metodologias analisadas. Os resultados correspondentes à metodologia de ELISA foram registrados na forma de rácio, que corresponde à razão entre os valores obtidos da densidade ótica (DO) de cada amostra e o valor de corte (CO) ou limiar de reatividade $(\mathrm{DO} / \mathrm{CO})$ para cada teste ELISA. Amostras com valores de rácio iguais ou superiores a 1,0 foram consideradas positivas e inferiores a 1,0 , negativas. Para as metodologias VDRL e IFI, foram inseridos os valores de intensidade da reação de cada amostra em cruzes “+”, variando de 1+ a 4+, sendo 4+ a resposta máxima da intensidade. Os resultados das amostras frente às metodologias de TR e RPR foram descritos como positivos (POS) e negativos (NEG). 
Para revalidação de cada amostra do painel foram adotados os seguintes critérios: volume mínimo de estoque de $10 \mathrm{~mL}$; reatividade em três testes ELISA, um teste VRDL e/ou um teste RPR, um teste TR e um teste confirmatório de IFI. Sendo assim, as amostras que cumpriram os critérios adotados foram consideradas positivas. Amostras com resultado não reagente em todas as metodologias foram consideradas negativas. Foram consideradas indeterminadas as amostras não inseridas nos parâmetros anteriores.

\section{RESULTADOS}

Das 172 amostras identificadas no painel positivo para sífilis, 19 (11\%) foram inicialmente excluídas por apresentarem volume inferior a $10 \mathrm{~mL}$, portanto, 153 amostras seguiram para as etapas subsequentes de revalidação.

O levantamento dos dados laboratoriais referente às análises realizadas no período de janeiro de 2011 a dezembro de 2015 permitiu a identificação de 46 produtos de diagnóstico in vitro para sífilis analisados pelo LSH/INCQS com LA satisfatório, assim distribuídos: 13 kits no ano de 2011, seis kits em 2012, oito kits em 2013, 11 kits em 2014 e oito kits em 2015. Vale ressaltar que o número de kits recebidos anualmente está relacionado com a demanda de produtos para obtenção de registro pela Anvisa. A distribuição deste total de kits por metodologia analisada foi a seguinte: ELISA (12 produtos), VDRL (oito produtos), RPR (cinco produtos), TR (13 produtos) e IFI (oito produtos) (Tabela 1).
A partir da seleção dos 46 kits satisfatórios, foram identificados 145 protocolos de registro de resultados de todas as 153 amostras pertencentes ao painel positivo para sífilis. Do total de 145 protocolos de trabalho analisados, 31 (21\%) envolveram a metodologia de ELISA, 29 (20\%) de VDRL, 19 (13\%) de RPR, 26 (18\%) de TR e 40 (28\%) de IFI distribuídos nos anos de 2011 a 2015 (Tabela 2).

Os resultados das amostras do painel positivo para sífilis obtidos nos 145 protocolos analisados frente às metodologias selecionadas foram inseridos em planilha Exce $^{\circledast}$. De acordo com os critérios de reatividade estabelecidos para revalidação das amostras, foi observado que, dentre as 153 amostras do painel positivo para sífilis, nenhuma apresentou resultado negativo nas metodologias analisadas. Um total de 118 amostras (77\%) apresentou resultado positivo para três testes ELISA com rácio $\geq 1$, um teste VDRL, um teste RPR e um teste IFI, cumprindo todos os critérios de reatividade sendo revalidadas como amostras positivas; 12 amostras (8\%) apresentaram resultado positivo para três testes ELISA com rácio $\geq 1$, um teste VDRL ou um teste $R P R$, um teste TR e um teste IFI, sendo também revalidadas como amostras positivas, totalizando um número de 130 amostras $(85 \%)$ com reatividade comprovada. Vinte e três amostras (15\%) apresentaram resultado negativo para duas ou mais metodologias sendo classificadas como amostras indeterminadas (Figura).

Dentre as 23 amostras classificadas como indeterminadas, três $(13 \%)$ apresentaram resultado positivo para as três metodologias de testes treponêmicos, que detectam anticorpos

Tabela 1. Distribuição do $\mathrm{n}^{\circ}$ de produtos de diagnóstico in vitro de sífilis analisados por ano e por metodologia (período 2011 - 2015 ).

\begin{tabular}{|c|c|c|c|c|c|c|}
\hline Metodologia & 2011 & 2012 & 2013 & 2014 & 2015 & Total \\
\hline ELISA & 5 & 1 & 3 & 1 & 2 & 12 \\
\hline VDRL & 2 & 2 & 2 & 1 & 1 & 8 \\
\hline RPR & 0 & 0 & 1 & 2 & 2 & 5 \\
\hline TR & 5 & 2 & 1 & 4 & 1 & 13 \\
\hline IFI & 1 & 1 & 1 & 3 & 2 & 8 \\
\hline TOTAL & 13 & 6 & 8 & 11 & 8 & 46 \\
\hline
\end{tabular}

Fonte: Elaborada pelos autores, 2020.

ELISA: ensaio imunoenzimático; VDRL: venereal disease research laboratory; RPR: rapid plasm reagin; TR: teste rápido imunocromatográfico; IFI: imunofluorescência indireta.

Tabela 2. Distribuição do número de protocolos de registro de resultados por ano e por metodologia de produtos de diagnóstico in vitro de sífilis analisados.

\begin{tabular}{|c|c|c|c|c|c|c|}
\hline Metodologia & 2011 & 2012 & 2013 & 2014 & 2015 & Total \\
\hline ELISA & 12 & 2 & 6 & 2 & 9 & 31 \\
\hline VDRL & 5 & 7 & 11 & 4 & 2 & 29 \\
\hline RPR & 0 & 0 & 5 & 4 & 10 & 19 \\
\hline TR & 8 & 4 & 3 & 9 & 2 & 26 \\
\hline IFI & 7 & 6 & 10 & 11 & 6 & 40 \\
\hline TOTAL & 32 & 19 & 35 & 30 & 29 & 145 \\
\hline
\end{tabular}

Fonte: Elaborada pelos autores, 2020.

ELISA: ensaio imunoenzimático; VDRL: venereal disease research laboratory; RPR: rapid plasm reagin; TR: teste rápido imunocromatográfico; IFI: imunofluorescência indireta. 


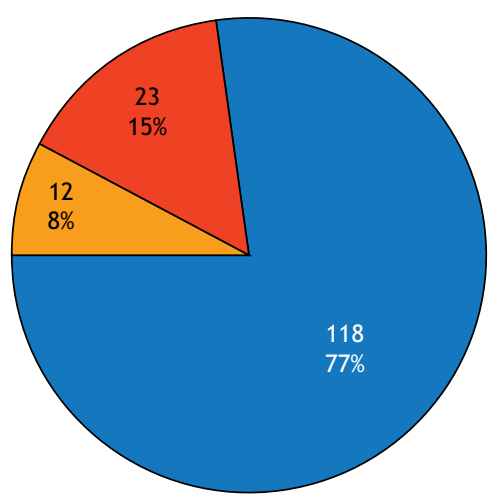

Amostras positivas para testes treponêmicos e não-treponêmicos

$\square$ Amostras positivas para testes treponêmicos e um não-treponêmico

$\square$ Amostras indeterminadas

Fonte: Elaborada pelos autores, 2020.

Figura. Distribuição dos resultados das amostras do painel positivo para sífilis revalidado.

específicos contra $T$. pallidum (ELISA, TR, IFI), e resultado negativo ou indeterminado para duas metodologias de testes não treponêmicos, aqueles que detectam anticorpos contra o material lipídico liberado pelas células danificadas em decorrência da sífilis (VDRL, RPR); 13 apresentaram resultado positivo para a IFI e resultado negativo para as metodologias ELISA e TR; cinco apresentaram resultado negativo para ELISA, TR, VDRL e/ ou RPR e duas amostras não apresentaram resultado para IFI (Tabela 3).

Em resumo, após a revalidação do painel positivo para sífilis composto de 153 amostras, 130 (85\%) foram confirmadas como positivas enquanto $23(15 \%)$ amostras passaram a ser classificadas como indeterminadas requerendo assim novas análises nas diferentes metodologias.

\section{DISCUSSÃO}

O percentual de $11 \%(19 / 172)$ das amostras excluídas em virtude do volume inferior a $10 \mathrm{~mL}$ se deve à grande demanda do laboratório para análise dos kits para diagnóstico da sífilis ao longo destes anos, principalmente nas análises automatizadas que requerem um volume maior de amostra para sua realização.

Foi possível observar após todo processo de revalidação das amostras do painel positivo para sífilis do LSH que, das 153 amostras iniciais, nenhuma foi descartada por ser considerada como amostra negativa e 23 amostras passaram a ser caracterizadas como amostras indeterminadas.

Dentre as amostras caracterizadas como indeterminadas, é possível sugerir sobre aquelas com resultado positivo em todos os testes treponêmicos e resultado negativo para os testes não treponêmicos que demonstram um padrão de resposta sorológica característica de infecção recente. Na literatura, este padrão de resposta na sífilis primária está muito bem descrito e ratificado ${ }^{5,6,14}$.
Tabela 3. Distribuição dos resultados das amostras indeterminadas na revalidação do painel positivo para sífilis.

\begin{tabular}{lc}
\hline Metodologia & $\begin{array}{c}\text { Amostras não } \\
\text { revalidadas }\end{array}$ \\
\hline VDRL e RPR & 3 \\
ELISA e TR & 13 \\
ELISA, TR, VDRL e/ou RPR & 5 \\
IFI & 2 \\
Total & 23
\end{tabular}

Fonte: Elaborada pelos autores, 2020

VDRL: venereal disease research laboratory; RPR: rapid plasm reagin; ELISA: ensaio imunoenzimático; TR: teste rápido imunocromatográfico; IFI: imunofluorescência indireta.

No caso das amostras caracterizadas como indeterminadas, que apresentaram resultado positivo para IFI, porém negativo para outros testes treponêmicos e negativo para um ou mais testes não treponêmicos, é possível sugerir que possuam um padrão de resposta sorológica característico de cicatriz sorológica. Considera-se cicatriz sorológica a persistência, após dois anos de acompanhamento pós-tratamento, da resposta sorológica aos testes não treponêmicos em títulos baixos (até 1:4) acompanhada de testes treponêmicos positivos ${ }^{14}$. Em cerca de $85 \%$ de pacientes com tratamento bem-sucedido, este perfil de resposta positiva para testes treponêmicos pode se manter durante vários anos ${ }^{15,16}$.

Todo processo produtivo apresenta etapas muito bem definidas e, dentre elas, está a etapa de validação anterior à implementação do produto bem como etapas que se seguem a esta para o controle da eficácia e qualidade do produto. 0 painel positivo para sífilis constituiu-se como produto a ser avaliado através da etapa de revalidação, sendo sua relevância fundamentada no tempo de uso destas amostras, que é superior a 10 anos, considerando que este painel foi constituído por amostras de plasma recebidas pelo LSH/INCQS entre os anos de 1996 e 2006. Vários estudos sobre a avaliação de produtos para diagnóstico da sífilis demonstraram a utilização de painéis constituídos por amostras provenientes de bancos de sangue e a importância de uma caracterização muito bem realizada através de testes treponêmicos e não treponêmicos de diferentes metodologias ${ }^{17,18}$.

\section{CONCLUSÕES}

Após a análise das 153 amostras que constituíam o painel sorológico positivo para sífilis, frente a 46 produtos com LA satisfatório, de cinco metodologias diferentes, foi possível revalidar o painel que passou a ser composto por 130 amostras verdadeiro positivas.

O painel sorológico positivo para sífilis revalidado permanecerá sendo instrumento essencial na análise prévia para fins de regularização dos kits para diagnóstico sorológico da sífilis. É uma ferramenta utilizada na análise de produtos com a finalidade de garantir resultados confiáveis e ampliar a capacidade analítica do LSH. 


\section{REFERÊNCIAS}

1. Souza EM. Há 100 anos, a descoberta do Treponema pallidum. An Bras Dermatol. 2005;80(5):547-8. https://doi.org/10.1590/\$0365-05962005000600017

2. Ministério da Saúde (BR). Boletim epidemiológico sífilis. Brasília: Ministério da Saúde; 2016.

3. Unemo M, Bradshaw CS, Hocking JS, Vries HJ, Francis SC, Mabey D et al. Sexually transmitted infections: challenges ahead. Lancet Infect Dis. 2017;17(8):e235-79. https://doi.org/10.1016/S1473-3099(17)30310-9

4. Ministério da Saúde (BR). Boletim epidemiológico sífilis. Brasília: Ministério da Saúde; 2018.

5. Avelleira JCR, Bottino G. Sífilis: diagnóstico, tratamento e controle. An Bras Dermatol. 2006;81(2):111-26. https://doi.org/10.1590/\$0365-05962006000200002

6. Contreras E, Zuluaga SX, Ocampo V. Sífilis: Ia gran simuladora. Infectio. 2008;12(2):120-7.

7. Ministério da Saúde (BR). Protocolo clínico e diretrizes terapêuticas para prevenção da transmissão vertical de HIV, sífilis e hepatites virais. Brasília: Ministério da Saúde; 2015.

8. Ministério da Saúde (BR). Diagnóstico da sífilis. Brasília: Ministério da Saúde; 2014.

9. Ministério da Saúde (BR). Portaria $N^{\circ} 158$, de 4 de fevereiro de 2016. Redefine o regulamento técnico de procedimentos hemoterápicos. Diário Oficial União. 5 fev 2016.

10. Adegoke AO, Akanni OE. Survival of Treponema pallidum in banked blood for prevention of syphilis transmission. N Am J Med Sci. 2011;3(7):329-32. https://doi.org/10.4297/najms.2011.3329

11. Agência Nacional de Vigilância Sanitária - Anvisa. Resolução RDC N ${ }^{\circ} 36$, de 26 de agosto de 2015. Dispõe sobre a classificação de risco, os regimes de controle de cadastro e registro eos requisitos de rotulagem e instruções de uso de produtos para diagnóstico in vitro,inclusive seus instrumentos e dá outras providências. Diário Oficial União. 27 ago 2015.

12. Fundação Oswaldo Cruz - Fiocruz. O que são painéis sorológicos? Rio de Janeiro: Fundação Oswaldo Cruz; 2014[acesso 8 jan 2018]. Disponível em: https://www.bio. fiocruz.br/index.php/perguntas-frequentes/70-perguntasfrequentes/perguntas-frequentes-reativos/228-o-que-saopaineis-sorologicos

13. Ministério da Saúde (BR). Portaria de consolidação $N^{\circ} 5$, de 28 de setembro de 2017. Consolidação das normas sobre as ações e os serviços de saúde do sistema único de saúde. Diário Oficial União. 3 out 2017.

14. Rotta O. Serological diagnosis of syphilis. An Bras Dermatol. 2005;80(3):299-302. https://doi.org/10.1590/S0365-05962005000300014

15. Peeling RW, Hook EW. The pathogenesis of syphilis: the great mimicker, revisited. J Pathol. 2006;208(2):224-32. https://doi.org/10.1002/path.1903

16. Stevenson J, Heath M. Syphilis and HIV infection: an update. Dermat Clin. 2006;24(4):497-507. https://doi.org/10.1016/j.det.2006.06.013

17. Mehra B, Bhattar S, Saxena S, Rawat D, Bhalla P. Evaluation of SD Bioline syphilis 3.0 for rapid diagnosis of syphilis: report from a regional sexually transmitted infection reference laboratory in North India. J Lab Physicians. 2016;8(1):36-40. https://doi.org/10.4103/0974-2727.176239

18. Bazzo ML, Motta LR, Rudolf-Oliveira RCM, Bigolin A, Golfetto L, Mesquita F et al. Evaluation of seven rapid tests for syphilis available in Brazil using defibrinated plasma panels. Sex Transm Infect. 2017;93(S4):S46-S50. https://doi.org/10.1136/sextrans-2017-053177

Agradecimentos

Este trabalho foi realizado com apoio da Coordenação de Aperfeiçoamento de Pessoal de Nível Superior-Brasil (CAPES) - Código de Financiamento 001.

\section{Contribuição dos autores}

Passo DCD - Concepção, planejamento (desenho do estudo), aquisição, análise, interpretação dos dados e redação do trabalho. Zamith HPS, Coelho MA - Concepção, planejamento (desenho do estudo), análise, interpretação dos dados e redação do trabalho. Vigo DC, Ribeiro AS, Passo RM - Aquisição dos dados. Todos os autores aprovaram a versão final do trabalho.

Os autores informam não haver qualquer potencial conflito de interesse com pares e instituições, políticos ou financeiros deste estudo.

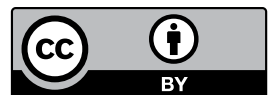

Esta publicação está sob a licença Creative Commons Atribuição 3.0 não Adaptada.

Para ver uma cópia desta licença, visite http://creativecommons.org/licenses/by/3.0/deed.pt_BR. 